\title{
Vena cava backflow and right ventricular stiffness in pulmonary arterial hypertension
}

\author{
J. Tim Marcus ${ }^{1}$, Berend E. Westerhof ${ }^{2,3}$, Joanne A. Groeneveldt ${ }^{2}$, \\ Harm Jan Bogaard ${ }^{2}$, Frances S. de Man $^{2}$ and Anton Vonk Noordegraaf ${ }^{2}$
}

Affiliations: ${ }^{1}$ Radiology and Nuclear Medicine, Amsterdam Cardiovascular Sciences, Amsterdam UMC, Vrije Universiteit Amsterdam, Amsterdam, The Netherlands. ${ }^{2}$ Pulmonary Medicine, Amsterdam Cardiovascular Sciences, Amsterdam UMC, Vrije Universiteit Amsterdam, Amsterdam, The Netherlands. ${ }^{3}$ Medical Biology, Section of Systems Physiology, Amsterdam Cardiovascular Sciences, Amsterdam UMC, University of Amsterdam, Amsterdam, The Netherlands.

Correspondence: J. Tim Marcus, Radiology and Nuclear Medicine, Amsterdam Cardiovascular Sciences, Amsterdam UMC, Vrije Universiteit Amsterdam, De Boelelaan 1117, 1081 HV Amsterdam, The Netherlands. E-mail: jt.marcusavumc.nl

@ERSpublications

In 62 patients with pulmonary arterial hypertension, timing of maximal backflow in the vena cava was in late diastole, during the right atrial contraction. The amount of backflow was associated with right ventricular stiffness. Stroke volume was reduced. http://bit.ly/2Y8YKg8

Cite this article as: Marcus JT, Westerhof BE, Groeneveldt JA, et al. Vena cava backflow and right ventricular stiffness in pulmonary arterial hypertension. Eur Respir J 2019; 54: 1900625 [https://doi.org/ 10.1183/13993003.00625-2019].

ABSTRACT Vena cava backflow is a well-recognised clinical hallmark of right ventricular failure in pulmonary arterial hypertension (PAH). Backflow may result from tricuspid regurgitation during right ventricular systole or from impaired right ventricular diastolic filling during atrial contraction. Our aim was to quantify the forward and backward flow in the vena cava and to establish the main cause in PAH.

In $62 \mathrm{PAH}$ patients, cardiac magnetic resonance measurements provided volumetric flows $\left(\mathrm{mL} \cdot \mathrm{s}^{-1}\right)$ in the superior and inferior vena cava; time integration of flow gave volume. The "backward fraction" was defined as the ratio of the backward and forward volumes in the vena cava, expressed as a percentage. Time of maximum vena cava backflow was expressed as a percentage of the cardiac cycle. Right ventricular volumes and aortic stroke volume were determined. Right heart catheterisation gave right ventricular and right atrial pressures. Right ventricular end-diastolic stiffness was determined with the single-beat method.

The median (interquartile range) backward fraction was $12 \%(3-24 \%)$ and it was $>20 \%$ in 21 patients. Maximum backflow occurred at near $90 \%$ of the cardiac cycle, coinciding with atrial contraction. The backward fraction was associated with maximal right atrial pressure (Spearman's $\mathrm{r}=0.77$ ), right ventricular end-diastolic stiffness $(\mathrm{r}=0.65)$ and right ventricular end-diastolic pressure $(\mathrm{r}=0.77)$, and was negatively associated with stroke volume $(\mathrm{r}=-0.61)$ (all $\mathrm{p}<0.001)$.

Significant backward flow in the vena cava was observed in a large group of PAH patients and occurred mostly during atrial contraction as a consequence of impaired right ventricular filling due to right ventricular diastolic stiffness. The backward flow due to tricuspid regurgitation was of significance in only a small minority of patients. 


\section{Introduction}

In pulmonary arterial hypertension (PAH), an increased afterload results in right ventricular dysfunction, and ultimately right ventricular failure and death $[1,2]$. Clinical signs of right ventricular failure include peripheral oedema and the distension of the jugular veins. Already described in the 19th century by the German physician Adolf Kussmaul, severe right ventricular failure can result in distension of the internal jugular vein during inspiration. In modern medicine, the sign of venous backflow of intravenous contrast injected during computed tomography angiography is a frequent first sign pointing towards right ventricular failure. The clinical importance of venous backflow lies not only in a potential loss of right ventricular efficiency (the movement of blood requires energy), but also in a worsening of renal function as a result of venous congestion $[3,4]$.

Despite the wide recognition of venous backflow as an important hallmark of right ventricular failure, still very little is known about its main determinants. While right ventricular blood may flow away from the pulmonary circulation during systole due to tricuspid regurgitation, an alternative explanation is ejection of blood into the venous system during right atrial contraction. Such reversal of flow could become particularly prominent when right ventricular diastolic filling is impaired due to increased right ventricular stiffness. While most physiological studies on right ventricular failure have focused on systolic right ventricular dysfunction [5], right atrial pressure, a surrogate marker of right ventricular stiffness, was of prognostic significance in multiple epidemiological studies [6-8]. The haemodynamic consequences of right ventricular diastolic stiffness are largely unknown, but could include the backflow of venous blood and venous congestion.

During the diastolic part of the cardiac cycle, the healthy right ventricle fills with an amount of blood that is approximately equal to the stroke volume. A low stroke volume is associated with poor prognosis $[9,10]$. The normal direction of flow towards the right ventricle is determined by small pressure differences between central venous, right atrial and right ventricular pressure $[11,12]$. In PAH, a backflow has been observed in the vena cava $[13,14]$. Reversal of flow requires energy, which can only be generated by either right ventricular contraction in the presence of tricuspid regurgitation $[15,16]$ or by right atrial contraction in a setting of impaired right ventricular filling due to stiffness. Irrespective of its cause, backflow will decrease cardiac efficiency, and may cause deleterious venous congestion of the kidneys, liver and gut. However, until now, the cause, degree and consequences of venous backflow have not been examined. In this study we used cardiovascular magnetic resonance (CMR) to quantify the magnitude and direction of flow in the vena cava, in order to determine the relation between vena cava backflow and right ventricular diastolic stiffness.

\section{Materials and methods}

\section{Patients}

We analysed data from the prospective registry of PAH patients seen at Amsterdam UMC, Vrije Universiteit Amsterdam (Amsterdam, The Netherlands). Patients routinely undergo CMR imaging, right heart catheterisation (RHC), 6-min walk testing and blood sampling at diagnosis and during follow-up. $\mathrm{CMR}$ and $\mathrm{RHC}$ were performed within a 2-day time interval. Patient inclusion occurred without prior knowledge of current disease severity, medication or RHC results. The Medical Ethics Review Committee of the Vrije Universiteit University Medical Center did not consider the current study to fall within the scope of Medical Research Involving Human Subjects (WMO) and an informed consent statement was not obtained (approval 2012288).

\section{CMR measurement of vena cava flow and right ventricular volumes}

The patients underwent a CMR investigation to assess cardiac function and blood flow in the vena cava. CMR was performed with a $1.5 \mathrm{~T}$ Avanto whole body scanner (Siemens Healthcare, Erlangen, Germany) equipped with a six-element phased-array body coil. Phase-contrast velocity quantification was performed during shallow continued breathing, using a gradient echo pulse sequence with through-plane velocity encoding and a velocity sensitivity of $150 \mathrm{~cm} \cdot \mathrm{s}^{-1}$. Triggering was retrospective, field of view $240 \times 320 \mathrm{~mm}^{2}$, matrix size $140 \times 256$, echo time $3.4 \mathrm{~ms}$, temporal resolution $22 \mathrm{~ms}$, flip angle $15^{\circ}$. In the superior vena cava, the orientation of the image plane was transverse (figure 1a), at the position of the right pulmonary artery. In the inferior vena cava, flow was measured at a position just below the right atrium and above the venous branches to the liver and other abdominal organs (figure $1 \mathrm{~b}$ ).

By integrating the forward and backward parts of the flow curve, the forward and backward volumes over the entire cardiac cycle were calculated for the superior and inferior vena cava. To assess which part of the backward volume was caused by tricuspid regurgitation and which part was caused by right atrial contraction, backward volumes were also separately determined during ventricular systole and diastole, respectively. Total forward and backward volumes were obtained by taking the respective volumes of the superior and inferior vena cava together. Finally, the vena cava backward fraction was determined by 

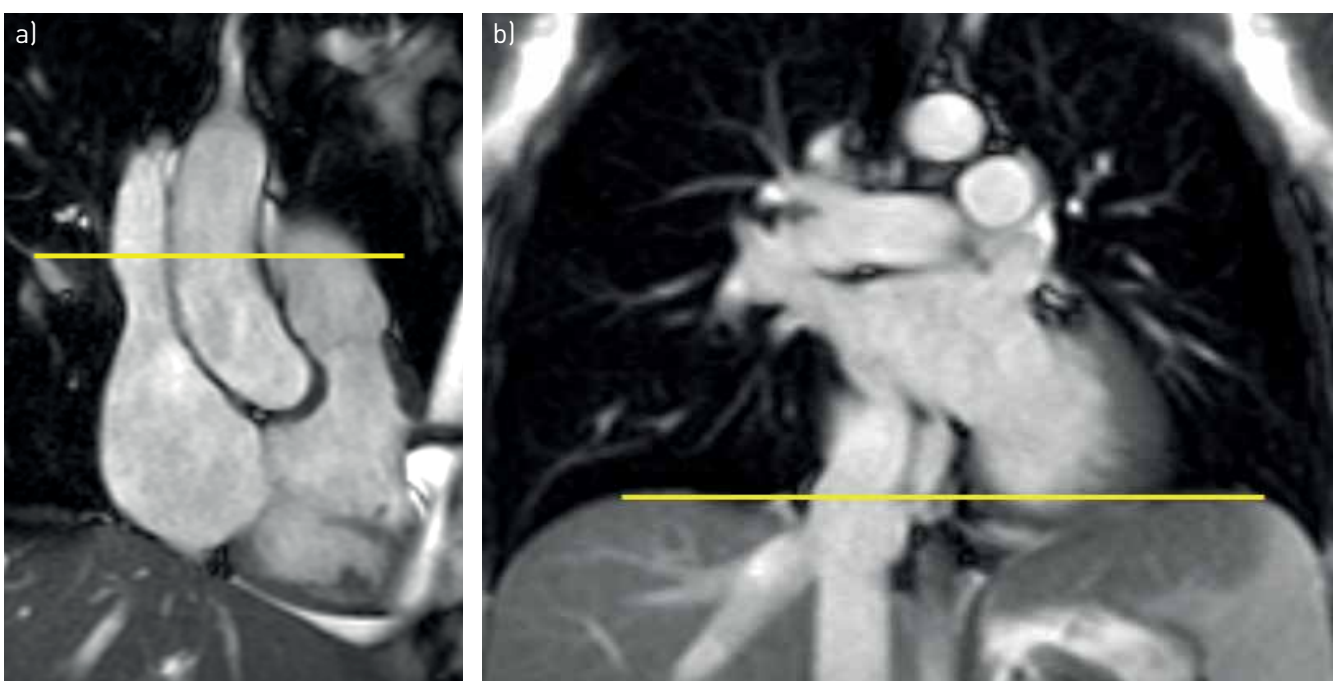

FIGURE 1 Planning of the cardiovascular magnetic resonance velocity quantification: imaging of the al superior vena cava and b) inferior vena cava. The yellow lines indicate the projections of the imaging planes used for through-plane velocity quantification.

dividing the total backward volume by the total forward volume per heartbeat and multiplying by 100 to give a percentage. The time of maximum backflow in the vena cava was expressed as a percentage of the cardiac cycle time.

Right ventricular end-systolic volume (RVESV) and right ventricular end-diastolic volume (RVEDV) were determined by applying short-axis coverage of the right ventricle by cine CMR. Maximal right atrial size was assessed by calculating right atrial cross-sectional area on the four-chamber cine CMR. The timeframe just before the opening of the tricuspid valve was selected for this calculation. MASS software (Medis, Leiden, The Netherlands) was used for these calculations. Stroke volume was obtained by integrating the aortic flow curve [17].

\section{Right heart catheterisation}

Under local anaesthesia, a balloon-tipped Swan-Ganz catheter (834F75; Edwards Lifesciences, Irvine, CA, USA) was inserted via the jugular vein and brought into position. Pulmonary arterial, right ventricular and right atrial pressures were recorded with a $1000 \mathrm{~Hz}$ sampling rate under constant ECG monitoring. From the right ventricular pressure curve, minimum pressure gave right ventricular begin-diastolic pressure (RVBDP), while the highest diastolic pressure, just before the onset of isovolumic contraction, gave right ventricular end-diastolic pressure (RVEDP). From the right atrial pressure curve, the maximal value, $\mathrm{RAP}_{\max }$, was determined.

\section{Single-beat pressure-volume analysis of right ventricular pressures}

To determine end-diastolic elastance $\left(E_{\text {ed }}\right)$, the three-point method was used $[18,19]$. The first $P V$ point was set at $V=0 \mathrm{~mL}, P=0 \mathrm{mmHg}$. The other two points were given by $V=\mathrm{RVESV}, P=\mathrm{RVBDP}$ and $V=$ RVEDV, $P=$ RVEDP, respectively. To avoid the effect of measurement offset errors due to the positioning of the right ventricular catheter pressure transducer, RVBDP was normalised at $1 \mathrm{mmHg}$, while RVEDP was calculated with the following formula: RVEDP normalised $=1+($ RVEDP-RVBDP). Through the three points, an exponential curve was fitted given by the formula $[18,20]: P=\alpha\left(\mathrm{e}^{V \beta}-1\right)$, where $P$ is the pressure $(\mathrm{mmHg}), \alpha$ is the curve-fitting constant $(\mathrm{mmHg}), \beta$ is the diastolic stiffness constant $\left(\mathrm{mL}^{-1}\right)$ and $V$ is the volume $(\mathrm{mL}) . E_{\text {ed }}$ was then calculated as the slope of this curve at end-diastolic volume $\left(\alpha \beta \mathrm{e}^{\beta E D V}\right)$ [19] and used as a measure of right ventricular stiffness $\left(\mathrm{mmHg} \cdot \mathrm{mL}^{-1}\right)$. Single-beat pressure-volume analyses were performed in Mathematica (Wolfram Research, Champaign, IL, USA).

\section{Statistics}

Normally distributed variables are presented as mean with standard deviation. Non-normally distributed variables are presented as median (interquartile range (IQR)). Spearman's rank-order correlation was applied to assess the associations between vena cava backward fraction, haemodynamics and right ventricular function. The level of significance used for testing was $\mathrm{p}<0.05$. The statistical analyses were performed using Prism version 7 (GraphPad, San Diego, CA, USA). 


\section{Results}

\section{Patient characteristics}

62 patients were included in this study (48\% idiopathic PAH, 21\% hereditary PAH, 21\% PAH secondary to connective tissue disease, $6 \%$ chronic thromboembolic pulmonary hypertension, $2 \% \mathrm{PAH}$ secondary to HIV and $2 \%$ pulmonary veno-occlusive disease). The majority of patients were female and the mean \pm SD age was $51 \pm 14$ years. Medication consisted of endothelin receptor antagonists, phosphodiesterase- 5 inhibitors, prostacyclins and calcium antagonist. Double therapy consisted mainly of endothelin receptor antagonists in combination with phosphodiesterase- 5 inhibitors; triple therapy included prostacyclins as well. Patient characteristics are summarised in table 1 . The mean \pm SD stroke volume from right ventricular volumes was $62 \pm 18 \mathrm{~mL}$ and was not different from the measurement obtained by aortic flow $(57 \pm 16 \mathrm{~mL} ; \mathrm{p}=0.128)$.

\section{Vena cava backflow}

Figure 2 shows the magnetic resonance imaging magnitude and velocity images of the flow measurement in the inferior vena cava. In systole, at a trigger delay of $101 \mathrm{~ms}$, the direction of blood velocity in the vena

\section{TABLE 1 Patient characteristics and measurements}

\begin{tabular}{|c|c|}
\hline \multicolumn{2}{|l|}{ General characteristics } \\
\hline Age years & $51 \pm 14$ \\
\hline \multicolumn{2}{|l|}{ Sex } \\
\hline Female & 49 \\
\hline Male & 13 \\
\hline 6MWD m & $459 \pm 151$ \\
\hline NT-proBNP ng. $L^{-1}$ & $363(122-1150)$ \\
\hline \multicolumn{2}{|l|}{ Disease type } \\
\hline Idiopathic/hereditary PAH & 43 \\
\hline PAH associated with connective tissue disease & 13 \\
\hline HIV-associated PAH & 1 \\
\hline Pulmonary veno-occlusive disease & 1 \\
\hline Chronic thromboembolic pulmonary hypertension & 4 \\
\hline \multicolumn{2}{|l|}{ Treatment at time of assessment } \\
\hline None & 12 \\
\hline Mono therapy & 11 \\
\hline Double therapy & 34 \\
\hline Triple therapy & 5 \\
\hline \multicolumn{2}{|l|}{ Right heart catheterisation } \\
\hline HR beats $\min ^{-1}$ & $75 \pm 11$ \\
\hline Stroke volume $\mathrm{mL}$ & $77 \pm 25$ \\
\hline mPAP mmHg & $48 \pm 12$ \\
\hline mRAP mmHg & $8 \pm 5$ \\
\hline $\mathrm{S}_{\mathrm{vO}_{2}} \%$ & $68 \pm 10$ \\
\hline PAWP mmHg & $9 \pm 3$ \\
\hline PVR dyn $\cdot \mathrm{s} \cdot \mathrm{cm}^{-5}$ & $518(362-1417)$ \\
\hline $\mathrm{CO} L \cdot \mathrm{min}^{-1}$ & $5.8 \pm 1.9$ \\
\hline \multicolumn{2}{|l|}{ Cardiac magnetic resonance imaging } \\
\hline HR beats $\cdot \mathrm{min}^{-1}$ & $75 \pm 13$ \\
\hline Stroke volume $\mathrm{mL}$ & $57 \pm 16$ \\
\hline RVEDV $\mathrm{mL}$ & $163 \pm 64$ \\
\hline RVESV mL & $101 \pm 64$ \\
\hline RVEF \% & $42 \pm 14$ \\
\hline Maximal RA size $\mathrm{cm}^{2}$ & $30 \pm 10$ \\
\hline LVEDV $\mathrm{mL}$ & $104 \pm 30$ \\
\hline LVESV mL & $40 \pm 18$ \\
\hline LVEF \% & $63 \pm 9$ \\
\hline
\end{tabular}

Data are presented as mean \pm SD or median (interquartile range) dependent on normal distribution, or as $\mathrm{n}$. 6MWD: 6-min walk distance; NT-proBNP: N-terminal pro-brain natriuretic peptide; PAH: pulmonary arterial hypertension; HR: heart rate; mPAP: mean pulmonary arterial pressure; mRAP: mean right atrial pressure; $\mathrm{S}_{\mathrm{vO}_{2}}$ : mixed venous oxygen saturation; PAWP: pulmonary arterial wedge pressure; PVR: pulmonary vascular resistance; CO: cardiac output; RVEDV: right ventricular end-diastolic volume; RVESV: right ventricular end-systolic volume; RVEF: right ventricular ejection fraction; RA: right atrium; LVEDV: left ventricular end-diastolic volume; LVESV: left ventricular end-systolic volume; LVEF: left ventricular ejection fraction. 

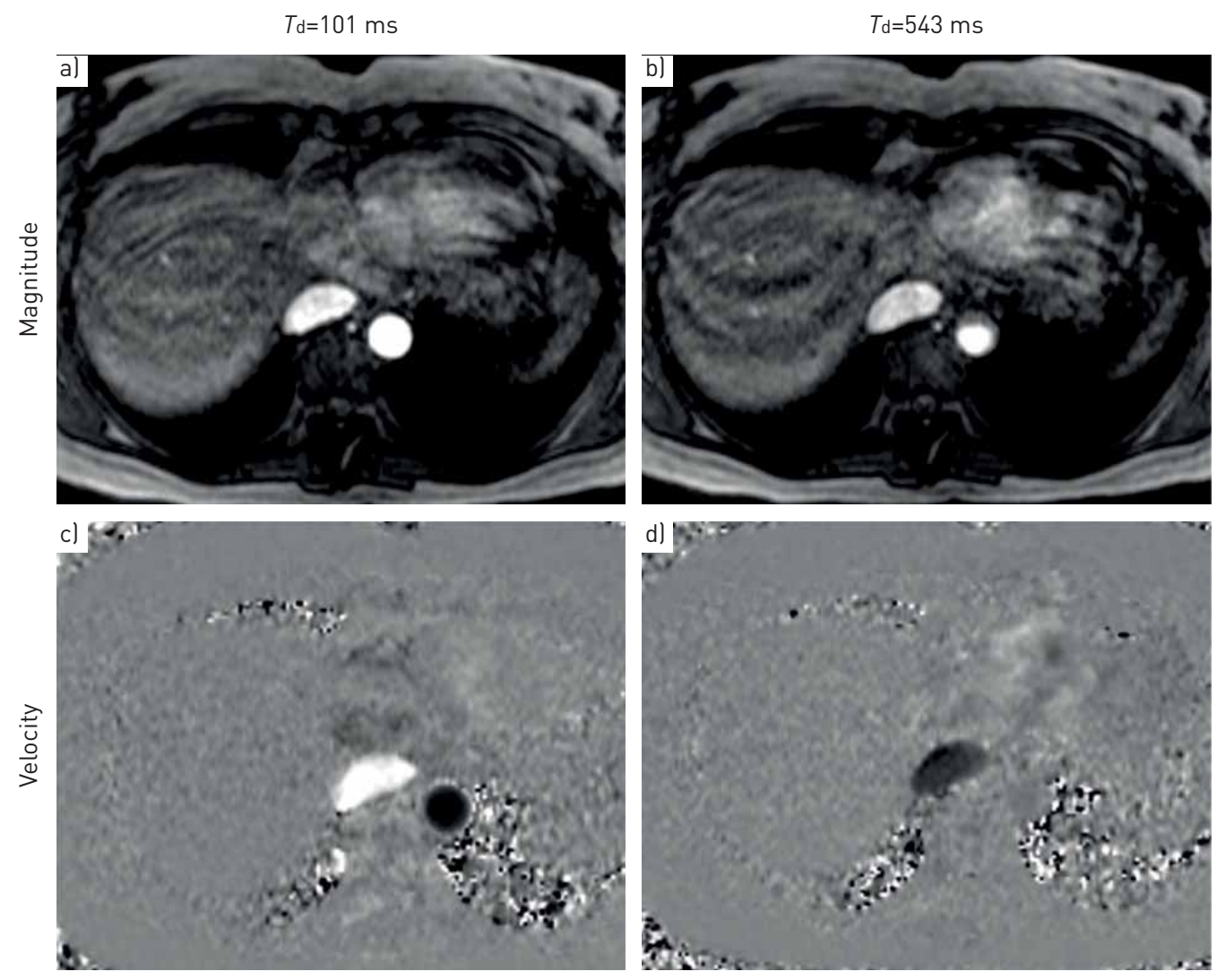

FIGURE 2 Cardiovascular magnetic resonance velocity quantification in the inferior vena cava: a, b) magnitude and $c, d$ ) velocity images of the inferior vena cava at two time-points in the cardiac cycle. $T_{\mathrm{d}}$ : trigger delay after the ECG R-wave. In the velocity images, "bright" indicates velocity towards the heart and "dark" indicates velocity away from the heart. a, c) At $T_{\mathrm{d}}=101 \mathrm{~ms}$, the velocity in the vena cava is directed towards the heart. $b$, d) At $T_{d}=543 \mathrm{~ms}$, the velocity in the vena cava has reversed and is now directed away from the heart.

cava is directed towards the heart (coded "bright"). In diastole, at a trigger delay of $543 \mathrm{~ms}$, the direction of vena cava blood velocity reverses to the backward direction away from the heart (coded "dark").

A diagram with simultaneous pressures in the pulmonary artery, right ventricle and right atrium in combination with vena cava flow measurements is given in figure 3. The CMR flow curves in the superior and inferior vena cava were aligned over the cardiac cycle using the ECG.

Maximum vena cava backflow was observed at a median (IQR) of 86\% (82-90\%) (superior vena cava) and 92\% (89-95\%) (inferior vena cava) of the cardiac cycle, coinciding with right ventricular diastole and atrial contraction. In two patients with severe tricuspid regurgitation, maximum backflow was observed in the systolic phase of the cardiac cycle. In the remaining 60 patients, maximum backflow occurred in the diastolic phase (atrial contraction) and amounted to a mean \pm SD of $93 \pm 11 \%$ of total backflow during the entire cardiac cycle. The median (IQR) backward fraction (ratio of backward to forward volume) was $12 \%$ (3-24\%) (figure 4). The backward fraction was significantly associated with $\mathrm{RAP}_{\max }(\mathrm{r}=0.77)$, RVEDP $(\mathrm{r}=0.77)$ and $E_{\text {ed }}(\mathrm{r}=0.65) \quad($ all $\mathrm{p}<0.001)$ (figure 4$)$. In addition, the backward fraction was negatively associated with stroke volume $(\mathrm{r}=-0.61)$ and right ventricular ejection fraction $(\mathrm{r}=-0.61)$ (table 2$)$. Only a weak negative correlation could be observed between the backward fraction and kidney function expressed as estimated glomerular filtration rate $(\mathrm{r}=-0.27 ; \mathrm{p}<0.05)$.

\section{Discussion}

From this study we conclude that, in a large group of PAH patients, backward flow in the vena cava can be observed in late diastole, which is closely associated with right ventricular diastolic stiffness. Tricuspid regurgitation, although present in almost all PAH patients, caused a maximal systolic backflow in the vena cava in only two patients. The bulk of backflow was observed during right atrial contraction.

The underlying mechanism of backflow is summarised in figure 5, which shows the pressures and flows during right atrial contraction. In a normal subject, the flow from the right atrium is in the forward 

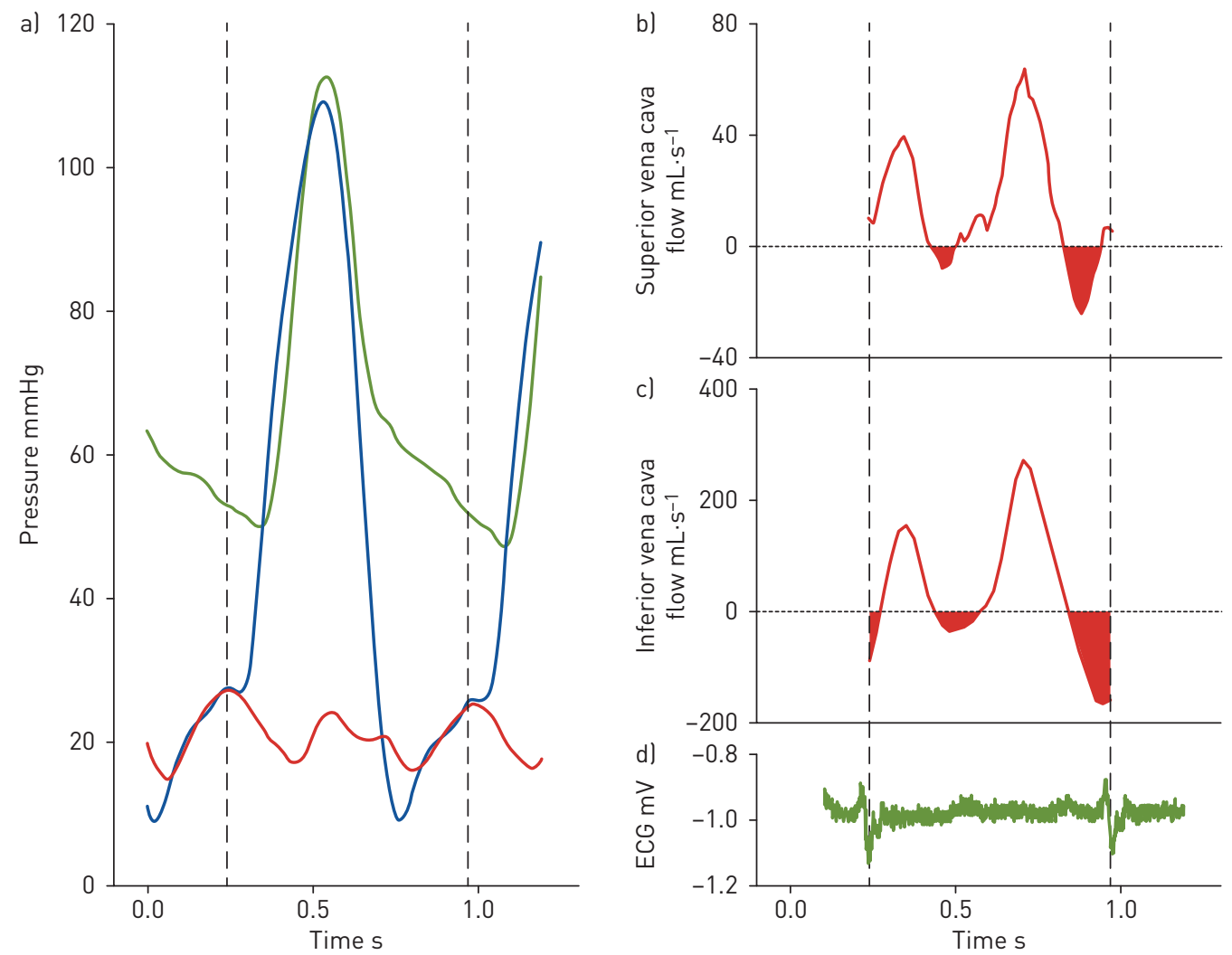

FIGURE 3 Pressure and flow measurements during the cardiac cycle. a) Simultaneous pressure recordings in a pulmonary arterial hypertension patient: pulmonary arterial (green), right ventricular (blue) and right atrial (red) pressure by right heart catheterisation. Note in the right ventricular pressure recording that after the beginning of diastole (lowest right ventricular pressure value), the right ventricular pressure increases quite steeply, which indicates that the right ventricular wall is stiff (diastolic dysfunction). b, c) For the same patient, magnetic resonance imaging-derived volumetric flow in the b) superior inferior vena cava and cl inferior vena cava. d) The timescale is synchronised using the ECG tracing. Flow towards the heart is positive and flow away from the heart is negative (backflow). The backward volumes are shown as red filled areas. The largest backward volume is late in the cardiac cycle, synchronous with the atrial contraction. In this patient, the total backward fraction is $40 \%$ ( $14 \%$ for the superior vena cava and $47 \%$ for the inferior vena cava). The backflow that occurs at the time when the pressure in the right ventricular rapidly increases, and that stops when the right ventricular pressure starts decreasing, is likely caused by tricuspid valve insufficiency. The low pressure in the right atrium at that moment is caused by the stretching of the right atrium by right ventricular contraction. The jet generated by the right ventricle through the insufficient tricuspid valve has a high velocity, but involves little volume, so that the pressure increase in the right atrium by the regurgitation is limited and the backflow into the vena cava is small.

direction, because the right ventricle is compliant and the RVEDP remains low. However, in a PAH patient with right ventricular diastolic dysfunction, the right ventricle is stiff and RVEDP is elevated. Blood flow will follow the largest downward pressure gradient and when the pressure in the vena cava is lower than in the right ventricle, flow will be partially directed back into the vena cava. As a consequence, venous return to the right ventricle is reduced, leading to a loss of right ventricular stroke volume. This chain of events is presented in figure 6. Based on the strongest associations with backflow, right ventricular stiffness increase and elevated RVEDP are given the central place in the process. Right ventricular volumes showed less tight relations and may be secondary effects. The relationship between the backward fraction and right atrial pressure and also maximal right atrial area suggests that the volume status of the patient may also be a factor influencing the amount of backflow.

Previously, echocardiographic measurements were used to show that an atrial reversal wave is present in hepatic venous flow [21]. In addition, pulmonary angiography showed reflux of contrast medium into the inferior vena cava and this reflux was associated with pulmonary hypertension [13] or with 30-day survival after pulmonary embolism [14]. The timing of backflow in the cardiac cycle was not determined in these angiography studies. We could demonstrate for the first time that vena cava backflow is mainly a phenomenon occurring during diastole and is therefore closely associated with right ventricular diastolic stiffness. The finding that the backward fraction was also positively associated with $E_{\mathrm{es}}$, while negatively 

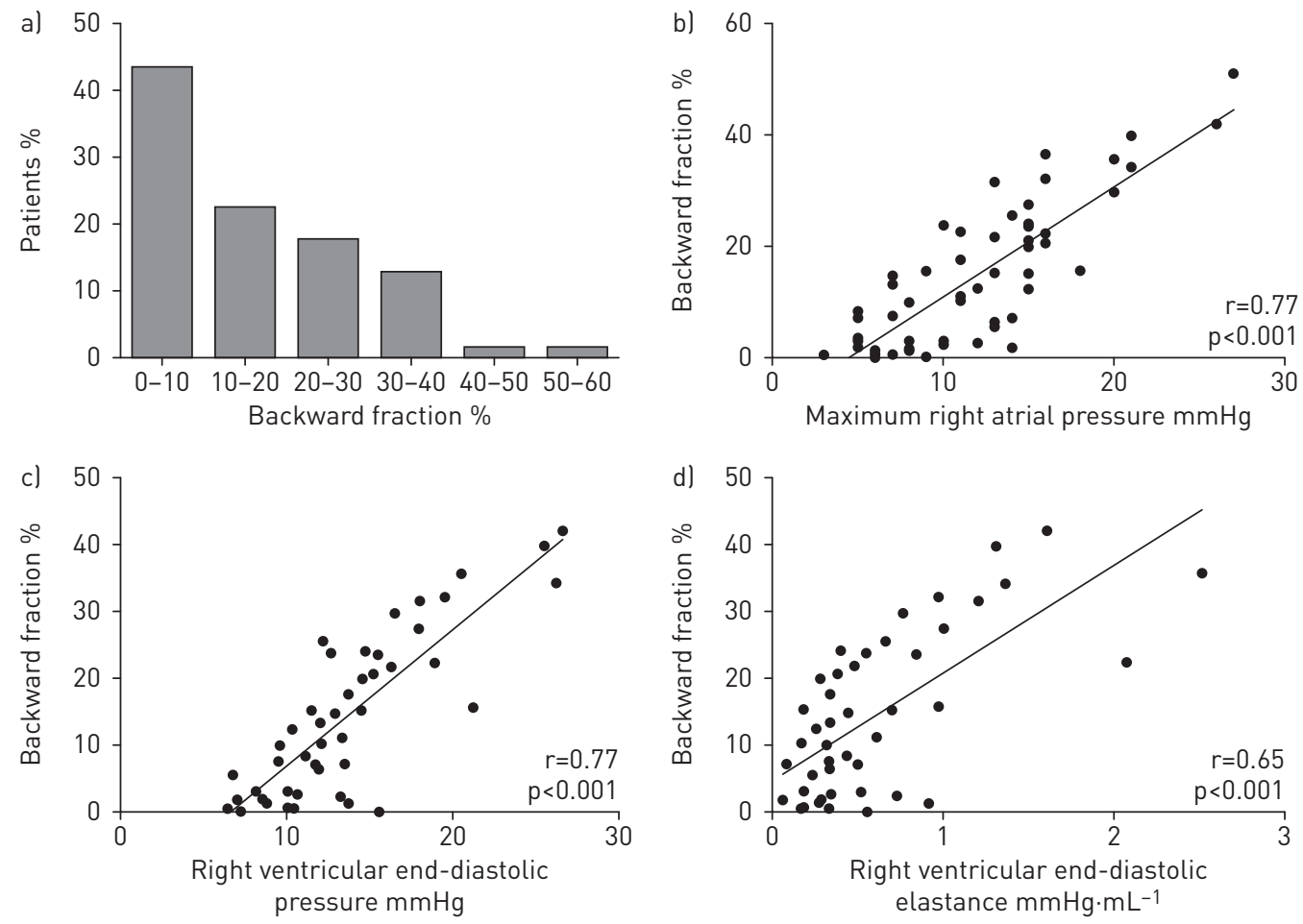

FIGURE 4 Association with the backward fraction. a) Backward fraction (defined as the ratio of backward volume and forward volume, expressed as a percentage) as distributed over the patient population. b-d) Backward fraction plotted versus b) maximal right atrial pressure, c) right ventricular end-diastolic pressure and d) right ventricular end-diastolic elastance as a measure of right ventricular stiffness.

associated with RVEF and stroke volume, can be clarified as follows. In the pressure overloaded right ventricle, hypertrophy is required to increase $E_{\mathrm{es}}$ in order to remain coupled to the load [5]. Hypertrophy implies wall thickening, which in turns entails a stiffer right ventricular and increased $E_{\text {ed. }}$ In this instance, RVEF and stroke volume are not measures of contractility.

\section{Clinical practice}

Our study introduces a new concept of backflow in the vena cava related to right atrial contraction. Instead of filling the right ventricle, the right atrium pumps a part of its volume back into the vena cava. An impact on stroke volume is suggested by the negative association between the backward fraction and

\begin{tabular}{lcc}
$\begin{array}{l}\text { TABLE } 2 \text { Associations between the backward fraction and clinical parameters } \\
\text { Parameter }\end{array}$ & Spearman's r & -value \\
\hline mPAP & 0.69 & $<0.001$ \\
Stroke volume & -0.61 & $<0.001$ \\
$\boldsymbol{E}_{\text {es }}$ & 0.61 & $<0.001$ \\
$\boldsymbol{E}_{\text {es }} / \boldsymbol{E}_{\text {a }}$ & -0.15 & 0.45 \\
RVEF & -0.61 & $<0.001$ \\
RVEDV & 0.35 & $<0.01$ \\
RVESV & 0.51 & $<0.001$ \\
Maximal RA size & 0.59 & $<0.001$ \\
In(NT-proBNP) & 0.71 & $<0.001$ \\
6MWD & -0.33 & $<0.01$ \\
eGFR & -0.27 & $<0.05$
\end{tabular}

mPAP: mean pulmonary arterial pressure; $E_{\mathrm{es}}$ : end-systolic elastance; $E_{\mathrm{a}}$ : arterial elastance; $E_{\mathrm{es}} / E_{\mathrm{a}}$ : ventricular-arterial coupling; RVEF: right ventricular ejection fraction; RVEDV: right ventricular end-diastolic volume; RVESV: right ventricular end-systolic volume; RA: right atrium; NT-proBNP: N-terminal pro-brain natriuretic peptide; 6MWD: 6-min walk distance; eGFR: estimated glomerular filtration rate. 
a)
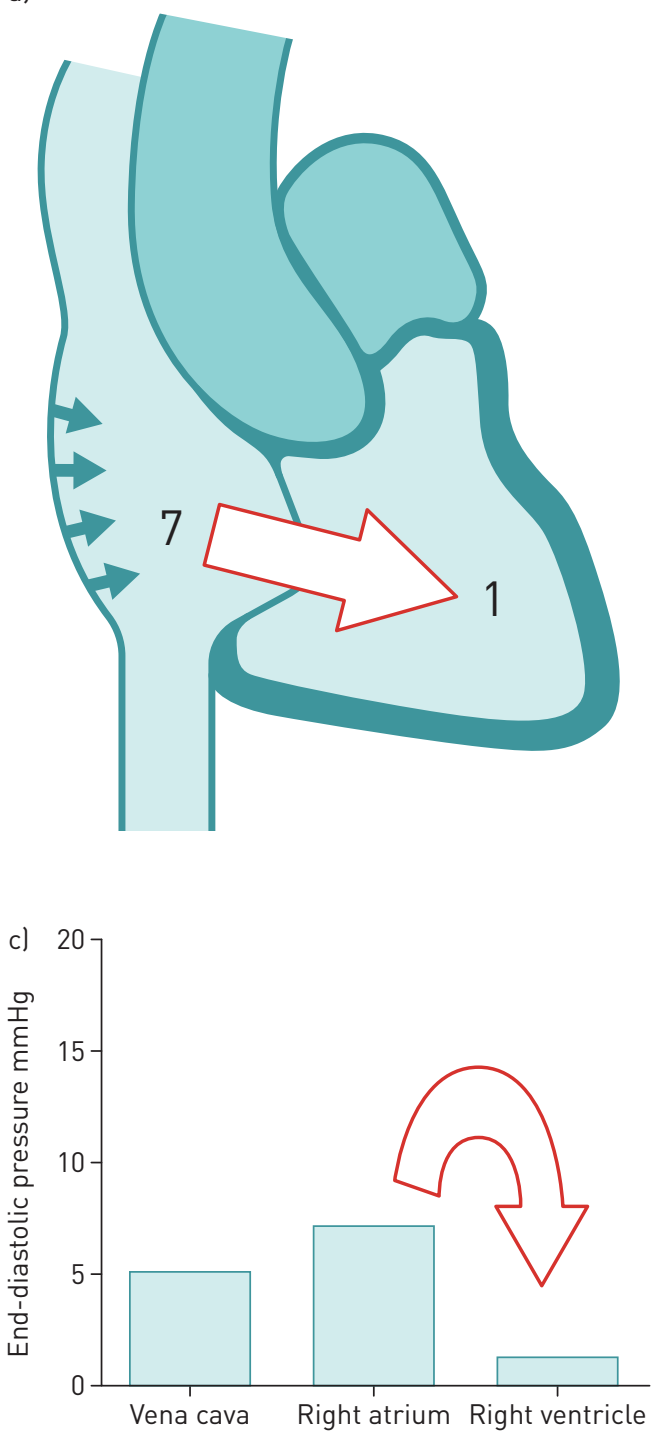

b)
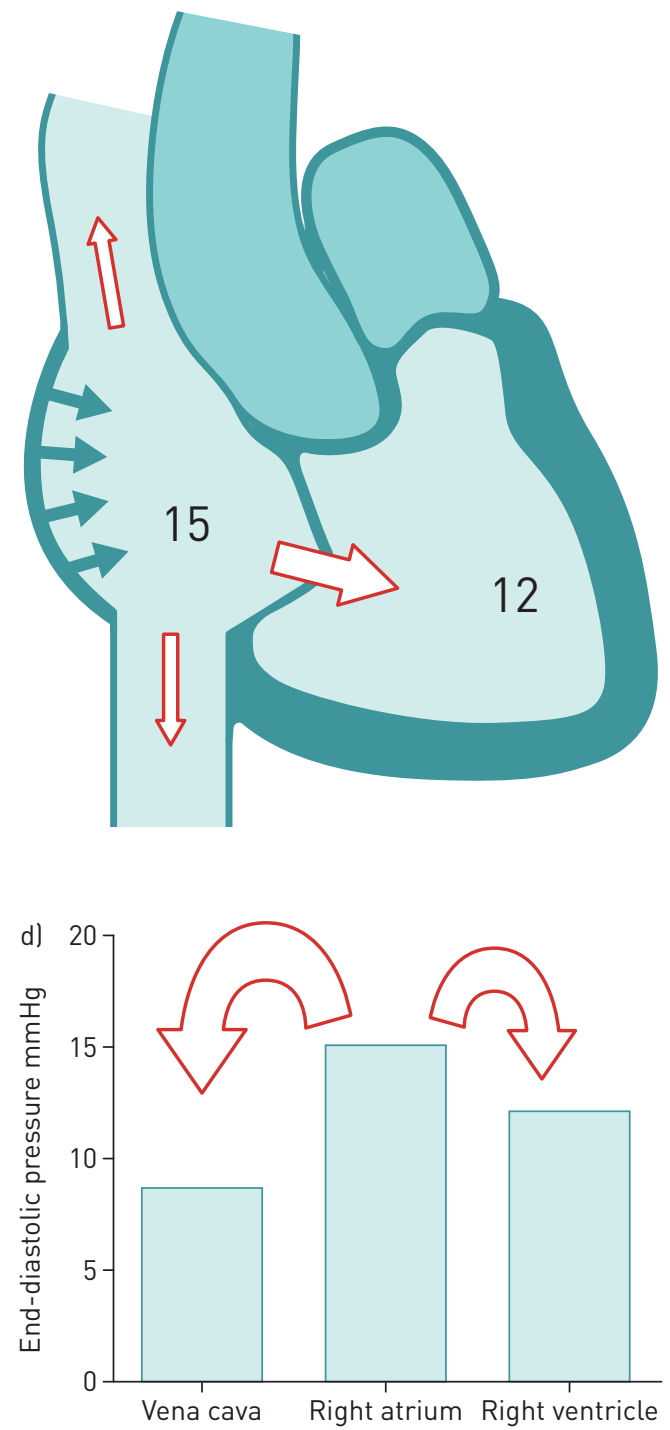

FIGURE 5 Pressures and flows in a normal and a stiff right ventricle. a, b) Cartoons of the right heart at end-diastole, during the atrial contraction. Numbers are representative pressures in $\mathrm{mmHg}$. c, d) Bar graphs indicating end-diastolic pressures in the vena cava, right atrium and right ventricle. a) A normal subject: because of the compliant right ventricular wall and the low right ventricular end-diastolic pressure (RVEDP), the right atrium ejects fully forward into the right ventricle (curved arrow in c). b) A patient with pulmonary arterial hypertension: due to the stiff right ventricular wall and the high RVEDP, the right atrium ejects partly in the reverse direction, back into the vena cava (curved arrows in d).

stroke volume. Through this mechanism, diastolic failure of the right ventricle is linked with systolic right ventricular failure, since loss of diastolic blood supply also implies loss of systolic stroke volume.

Therefore, this study demonstrates the potential detrimental effect of increased right ventricular diastolic stiffness on right ventricular systolic adaptation. Previous studies have shown that stiffening of the right ventricle is not just the consequence of hypertrophy, but is partially mediated by increased collagen deposition and hypophosphorylation of the giant sarcomeric protein titin $[18,19,22]$. Currently, no therapeutic strategies are available that directly target the right ventricle [23]. Our current study delineates once again the potential clinical importance of right ventricular diastolic stiffness in PAH and efforts should be made to obtain further insights into the pathophysiological mechanisms, possibly giving impetus to novel treatments.

\section{Limitations}

In our study, only associations were studied; further research is necessary to establish cause and effect relations. Another limitation is the lack of pressure recordings in the vena cava. The relative levels of the 
FIGURE 6 Flowchart: from right ventricular end-diastolic stiffness to vena cava backflow. This flowchart shows the proposed mechanism explaining how right ventricular stiffness leads to vena cava backflow and decreased right ventricular stroke volume.

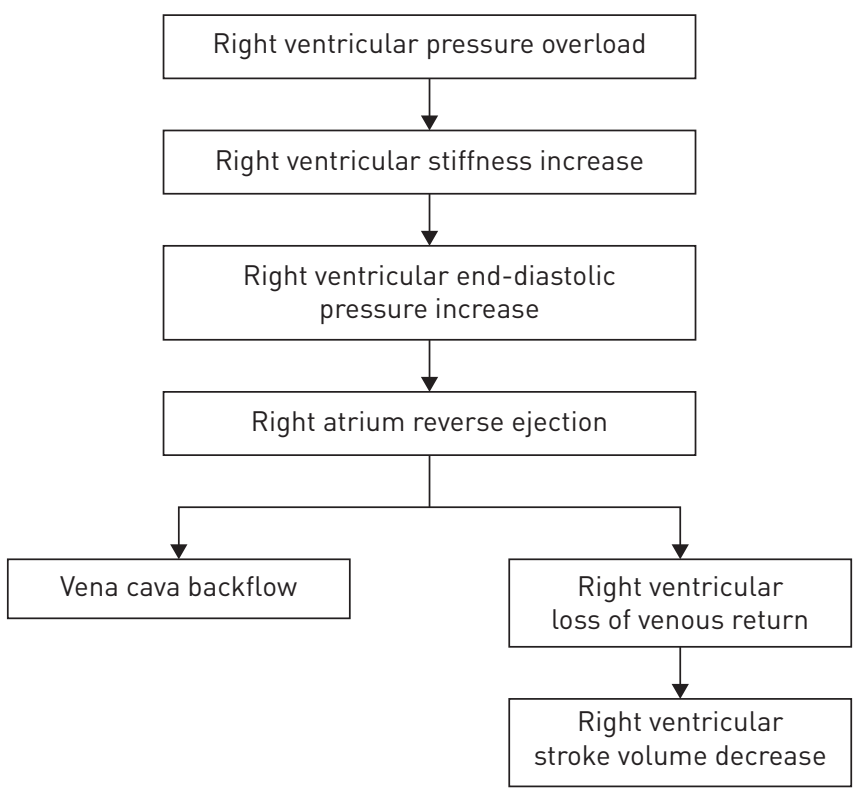

pressure in the vena cava and the pressure in the right ventricle will determine which fraction of blood is reversed by right atrial contraction.

Finally, although backflow could contribute to venous congestion [4], only a weak association could be observed between the backward fraction and kidney function in our study. This could possibly be explained by the fact that altered estimated glomerular filtration rate levels can be a reflection of both forward and backward failure. Unfortunately, levels of $\gamma$-glutamyl transpeptidase and alkaline phosphatase were determined in only a small percentage of patients, limiting correlation analyses. From our data it was not possible to determine to influence of the volume status on backflow. Future studies may investigate the effect of diuretics on the backward fraction.

\section{Conclusions and perspectives}

In the PAH patients of this study, the timing of maximal backflow in the vena cava was in late diastole, during the right atrial contraction. The backward fraction was associated with right ventricular stiffness. Because the right ventricle loses part of its venous return, the right ventricular stroke volume is reduced.

It is generally assumed that vena cava backflow is caused by tricuspid valve insufficiency and thus would occur during systole. In the PAH patients of this study, the vena cava backflow was mainly during atrial contraction and was associated with right ventricular end-diastolic stiffness.

The vena cava backflow limits the venous return to the right ventricle, reducing right ventricular stroke volume. The vena cava backflow may also contribute to venous congestion in these patients. Considerable differences in backflow are seen between patients. Further research may elucidate the causes of backflow in the individual patient.

The clinical implications of our findings remain to be determined. Our report contributes to an improved understanding of right heart inefficiency, a previously documented phenomenon associated with progressive right ventricular failure [24]. Right heart inefficiency in PAH is likely partly based on the cellular and molecular changes characteristic of heart failure, but also on mechanical inefficiency. Previously recognised contributors to mechanical inefficiency in right heart failure are post-systolic contraction [25] and tricuspid insufficiency. Here, we demonstrate the contribution of right atrial contraction-related vena cava backflow to mechanical inefficiency. As mechanical inefficiency may contribute to the progression of right ventricular failure, interventions to restore efficiency have been proposed, including right ventricle-left ventricle resynchronisation therapy and tricuspid valve repair. Further research is needed to investigate the pathophysiological causes of right ventricular diastolic stiffness in order to develop novel treatment strategies directly targeting the right ventricle.

Conflict of interest: J.T. Marcus reports personal fees for consultancy from Actelion Pharmaceuticals, outside the submitted work. B.E. Westerhof reports grants from the Netherlands Organization for Scientific Research, during the conduct of the study. J.A. Groeneveldt has nothing to disclose. H.J. Bogaard has nothing to disclose. F.S. de Man has 
nothing to disclose. A. Vonk Noordegraaf reports personal fees for lectures from Actelion and MSD, outside the submitted work.

Support statement: B.E. Westerhof and J.A. Groeneveldt were supported by NWO-VICI (918.16.610). H.J. Bogaard was supported by the Netherlands CardioVascular Research Initiative (CVON-2012-08 PHAEDRA, CVON-2017-10 DOLPHIN-GENESIS). A. Vonk Noordegraaf and F.S. de Man were supported by the Netherlands CardioVascular Research Initiative (CVON-2012-08 PHAEDRA, CVON-2017-10 DOLPHIN-GENESIS) and the Netherlands Organization for Scientific Research (NWO-VENI: 916.14.099, NWO-VIDI: 917.18.338, NWO-VICI: 918.16.610).

\section{References}

1 Galiè N, Humbert M, Vachiery JL, et al. 2015 ESC/ERS Guidelines for the diagnosis and treatment of pulmonary hypertension: The Joint Task Force for the Diagnosis and Treatment of Pulmonary Hypertension of the European Society of Cardiology (ESC) and the European Respiratory Society (ERS). Endorsed by: Association for European Paediatric and Congenital Cardiology (AEPC), International Society for Heart and Lung Transplantation (ISHLT). Eur Respir J 2015; 46: 903-975.

2 Vonk-Noordegraaf A, Haddad F, Chin KM, et al. Right heart adaptation to pulmonary arterial hypertension: physiology and pathobiology. J Am Coll Cardiol 2013; 62: D22-D33.

3 Mullens W, Abrahams Z, Francis GS, et al. Importance of venous congestion for worsening of renal function in advanced decompensated heart failure. J Am Coll Cardiol 2009; 53: 589-596.

4 Testani JM, Khera AV, St John Sutton MG, et al. Effect of right ventricular function and venous congestion on cardiorenal interactions during the treatment of decompensated heart failure. Am J Cardiol 2010; 105: 511-516.

5 Vonk Noordegraaf A, Westerhof BE, Westerhof N. The relationship between the right ventricle and its load in pulmonary hypertension. J Am Coll Cardiol 2017; 69: 236-243.

6 D’Alonzo GE, Barst RJ, Ayres SM, et al. Survival in patients with primary pulmonary hypertension. Results from a national prospective registry. Ann Intern Med 1991; 115: 343-349.

7 Benza RL, Miller DP, Gomberg-Maitland M, et al. Predicting survival in pulmonary arterial hypertension: insights from the Registry to Evaluate Early and Long-Term Pulmonary Arterial Hypertension Disease Management (REVEAL). Circulation 2010; 122: 164-172.

8 Weatherald J, Boucly A, Chemla D, et al. Prognostic value of follow-up hemodynamic variables after initial management in pulmonary arterial hypertension. Circulation 2018; 137: 693-704.

9 van Wolferen SA, van de Veerdonk MC, Mauritz GJ, et al. Clinically significant change in stroke volume in pulmonary hypertension. Chest 2011; 139: 1003-1009.

10 van de Veerdonk MC, Kind T, Marcus JT, et al. Progressive right ventricular dysfunction in patients with pulmonary arterial hypertension responding to therapy. J Am Coll Cardiol 2011; 58: 2511-2519.

11 Brecher GA. Cardiac variations in venous return studied with a new bristle flowmeter. Am J Physiol 1954; 176 423-430.

12 Wexler L, Bergel DH, Gabe IT, et al. Velocity of blood flow in normal human venae cavae. Circ Res 1968; 23: 349-359.

13 Aviram G, Cohen D, Steinvil A, et al. Significance of reflux of contrast medium into the inferior vena cava on computerized tomographic pulmonary angiogram. Am J Cardiol 2012; 109: 432-437.

14 Bach AG, Nansalmaa B, Kranz J, et al. CT pulmonary angiography findings that predict 30-day mortality in patients with acute pulmonary embolism. Eur J Radiol 2015; 84: 332-337.

15 Arsalan M, Walther T, Smith RL 2nd, et al. Tricuspid regurgitation diagnosis and treatment. Eur Heart J 2017; 38: 634-638.

16 Rivera JM, Vandervoort PM, Mele D, et al. Quantification of tricuspid regurgitation by means of the proximal flow convergence method: a clinical study. Am Heart J 1994; 127: 1354-1362.

17 Mauritz GJ, Marcus JT, Boonstra A, et al. Non-invasive stroke volume assessment in patients with pulmonary arterial hypertension: left-sided data mandatory. J Cardiovasc Magn Reson 2008; 10: 51.

18 Rain S, Handoko ML, Trip P, et al. Right ventricular diastolic impairment in patients with pulmonary arterial hypertension. Circulation 2013; 128: 2016-2025.

19 Trip P, Rain S, Handoko ML, et al. Clinical relevance of right ventricular diastolic stiffness in pulmonary hypertension. Eur Respir J 2015; 45: 1603-1612.

20 Jaber WA, Lam CS, Meyer DM, et al. Revisiting methods for assessing and comparing left ventricular diastolic stiffness: impact of relaxation, external forces, hypertrophy, and comparators. Am J Physiol Heart Circ Physiol 2007; 293: H2738-H2746.

21 Nagueh SF, Kopelen HA, Zoghbi WA. Relation of mean right atrial pressure to echocardiographic and Doppler parameters of right atrial and right ventricular function. Circulation 1996; 93: 1160-1169.

22 Rain S, da Silva Goncalves Bos D, Handoko ML, et al. Protein changes contributing to right ventricular cardiomyocyte diastolic dysfunction in pulmonary arterial hypertension. J Am Heart Assoc 2014; 3: e000716.

23 Westerhof BE, Saouti N, van der Laarse WJ, et al. Treatment strategies for the right heart in pulmonary hypertension. Cardiovasc Res 2017; 113: 1465-1473.

24 Wong YY, Ruiter G, Lubberink M, et al. Right ventricular failure in idiopathic pulmonary arterial hypertension is associated with inefficient myocardial oxygen utilization. Circ Heart Fail 2011; 4: 700-706.

25 Marcus JT, Gan CT, Zwanenburg JJ, et al. Interventricular mechanical asynchrony in pulmonary arterial hypertension: left-to-right delay in peak shortening is related to right ventricular overload and left ventricular underfilling. J Am Coll Cardiol 2008; 51: 750-757. 